'Petrom R.A., Institute for Research and Technology, 29 Culturii Boulevard, 2150 Campina, Romania

2 Ploiesti University

\title{
INTRODUCTION
}

Simulation of improved recovery processes from gas condensate and volatile oil reservoirs is significantly affected by the match of experimental PVT data. Therefore, a proper characterization of the mixture composition and the tuning of the equation of state used are crucial for the accuracy of the reservoir model.

Cubic equations of state (EOS) are well known to represent a very useful tool in calculating phase equilibrium, but they do not always succeed to describe with sufficient accuracy the behavior of natural hydrocarbon systems; the differences between the calculated values and actual values obtained from PVT experiments can influence to a great extent the results of prediction calculations. Some deviations from experimental values, of the results of the simulating models incorporating EOS, in a predictive manner, if the binary interaction coefficients (BIC) are not adjusted, are due both to the semi-empirical nature of the EOS, and to the uncertainties and approximations in characterization of the $\mathrm{C} 7+$ fraction.

The modem trend is to minimize the sum of the squared deviations between the results of the EOS-based models and those of the PVT tests, through application of the non-linear regression [1,2,3].

This paper presents an original approach in addressing this problem by the use of an efficient regression algorithm and of an associated computer program, designated as REGPVT [4]. A new, rigorous generalized quadrature method is developed for the characterization of the continuous portion of the mixture, which can be applied to any distribution function. The $\mathrm{C} 7+$ fraction is described by a continuous (semi-infinite or truncated) distribution function. The methods has been successful applied to several gas condensate systems.

\section{THE OPTIMIZATION PROBLEM}

By considering a set of experimental data whose values shall be given as accurately as possible through the mathematical model based on an EOS, the aim is to minimize the function:

$$
f(x)=\sum_{i=1}^{n e} r_{i}^{2}
$$

where $x=\left(x_{1}, x_{2}, \ldots x_{n r}\right)$ is the vector of the regression variables, $n r$ is the number of regression variables and ne is the number of experimental values (ne>nr).

The $r_{i}(x)$ functions represent the relative deviations of calculated values from experimental values, affected by the weight associated to each experimental point. The minimization of the objective function $f(x)$ represents a non-linear programming problem, namely, a constrained optimization problem. The optimization problem can be written as:

$$
\min f(\bar{x})=\vec{r}(\bar{x})^{\top} \cdot \vec{r}(\bar{x})
$$

The quadratic model at $x=x_{k}+\Delta x_{k}$, of the objective function $f$ (in the sense of the least squares) is [3]:

$$
q_{k}(\vec{x})=\vec{r}_{k}{ }^{\top} \vec{r}_{k}+2 \Delta \vec{x}_{k}{ }^{\top} J_{k}{ }^{\top} \vec{r}_{k}+\Delta \vec{x}_{k}{ }^{\top}\left(J_{k}{ }^{\top} J_{k}+S_{k}\right) \Delta \vec{x}_{k}
$$

where $J_{k}$ is the Jacobian matrix of elements: $J\left(\vec{x}_{k}\right)_{i j}=\frac{\partial r_{j}\left(\vec{x}_{k}\right)}{\partial\left(\vec{x}_{k}\right)_{j}}$, and $\mathbf{S}\left(\vec{x}_{k}\right)=\sum_{i=1}^{n e} r_{i}\left(\vec{x}_{k}\right) \nabla^{2} r_{i}\left(\vec{x}_{k}\right)$

By applying the Newton method, the following iterative scheme will result:

$$
\left(\mathbf{J}_{k}^{\top} \mathbf{J}_{k}+\mathbf{s}_{k}\right)\left(\vec{x}_{k}-\vec{x}_{k}\right)=-\mathbf{J}_{k}{ }^{\top} \vec{r}_{k}
$$


If the second order derivatives included in the expression of the Hessian matrix $\mathbf{H}_{k}=\left(\mathbf{J}_{k}{ }^{\top} \mathbf{J}_{k}+\mathbf{S}_{k}\right)$ are neglected, the method is of the Gauss-Newton type:

$$
\mathbf{J}_{k}^{\top} \mathbf{J}_{k}\left(\overrightarrow{\mathbf{x}}_{k+1}-\overrightarrow{\mathbf{x}}_{k}\right)=-\mathbf{J}_{k}{ }^{\top} \vec{r}_{k}
$$

The linear system of equations can be written as:

$$
\sum_{j=1}^{n r} \sum_{k=1}^{n e} \frac{\partial r_{k}}{\partial x_{i}} \frac{\partial r_{k}}{\partial x_{j}} \Delta x_{j}=-\sum_{k=1}^{n e} r_{k}\left(x_{i}\right) \frac{\partial r_{k}}{\partial x_{i}} ; i=1, n
$$

The $\Delta x$ solution of the system represents the search direction for the minimum of the objective function at the current iteration level. The elements of the Jacobian matrix are numerically evaluated through finite differences. Approximation of the derivatives requires the $n r$-fold repetition of the phase equilibrium calculation sequence. That is why the time associated with the numerical derivation represents the most important part of the total calculation time.

The REGPVT program comprises the following options for the experimental data: saturation pressure and related density ("basic PVT match"), volumetric values for the constant composition expansion (CCE), for the constant volume depletion (CVD) and for the differential expansion; data regarding multistage separation, and "swelling test" data. The global set of regression parameters includes the CBI and the $\Omega_{\mathrm{a}}$ and $\Omega_{\mathrm{b}}$ parameters of the EOS for methane and for the pseudo-components.

Within the program, the phase equilibrium calculations are based on the generalized form of the cubic EOS [1], particularized for the Peng-Robinson (PR) or the Soave-Redlich Kwong (SRK) EOS. The program is structured in such a manner as to be adapted, with minimal changes, to any EOS.

The dew point pressures have been calculated using the MVNR method (PX or PY iterations), while the flash calculations have been performed using the ACSS/MVNR-InK methods, the program being provided with options to extrapolate the results of previously performed flash calculations [4]. Also a version of the program has been developed, to incorporate a rapid phase equilibrium calculation method with a reduced number of independent variables $[4,5,6]$.

\section{THE LEVENBERG-MARQUARDT METHOD FOR REGRESSION ON PVT DATA}

The quadratic approximation for the $f(x)$ function is valid only within a $\Delta$ vicinity. This requires the setting up of a limit value for $\Delta x$; so, the optimization problem will be [7]:

$$
\begin{aligned}
& \min \left\{q_{k}(x):\|D \Delta x\| \leq \Delta\right\} \\
& q(\Delta \vec{x})=\|f(\vec{x})+\nabla f(\vec{x}) \Delta \vec{x}\|
\end{aligned}
$$

If:

$$
D=\operatorname{diag}\left(d_{1}, d_{2}, \ldots, d_{n}\right)
$$

$x_{k+1}$ will be located within the hyper-ellipsoid:

$$
E=\{\Delta \mathbf{x}:\|\mathbf{D} \Delta \mathbf{x}\| \leq \Delta\}
$$

The elements of the matrix $D$ are given by [7]:

$$
d_{i}{ }^{(k)}=\max \left\{d_{i}{ }^{(k-1)},\left\|\partial_{i} f\left(\vec{x}_{k}\right)\right\|\right\} ; i=1, n r ; k \geq 1 \text { and } d_{i}{ }^{(0)}=\left\|\partial_{i} f\left(\vec{x}_{0}\right)\right\| ; i=1, n r
$$

The hyper-ellipsoid represents the "trust region" where the local quadratic model $q_{k}$ stands valid for the $f$ function, given by the relation (3). By calculating the elements of the D matrix using the relation (11), the impact of the perturbation of each regression variable, on the change in the value of the objective function during the iterative process is being taken into consideration.

The Levenberg-Marquardt method consists in going through the following steps:

1. For a given $\Delta_{k}>0$, calculate $\lambda_{k} \geq 0$ to the effect that if:

$$
\left(\mathbf{J}_{k}{ }^{\top} \mathbf{J}_{k}+\lambda_{k} \mathbf{D}_{k}{ }^{\top} \mathbf{D}_{k}\right) \Delta \overrightarrow{\mathbf{x}}_{k}=-\mathbf{J}_{k}{ }^{\top} \overline{\boldsymbol{T}}_{\mathrm{k}}
$$

then either $\lambda_{k}=0$ and $\left\|\mathbf{D}_{k} \Delta \overline{\mathbf{x}}_{k}\right\| \leq \Delta_{k}$ or $\lambda_{k}>0$ and $\left\|\mathbf{D}_{k} \Delta \vec{x}_{k}\right\|=\Delta_{k}$.

2. If $\left\|f\left(\vec{x}_{k}+\Delta \vec{x}_{k}\right)\right\|<\left\|f\left(\vec{x}_{k}\right)\right\|$, then $x_{k+1}=x_{k}+\Delta x_{k}$ and $J_{k+1}$ are evaluated. Otherwise $x_{k+1}=x_{k}$ and $J_{k+1}=J_{k}$.

3. Calculate $\Delta_{k+1}$ and $D_{k+1}$.

The calculation of the vector elements determining the search direction of the optimum merely requires the solution of a linear system and of a non-linear equation. A characteristic feature of the LevenbergMarquardt method is the concurrent change, with a minimum calculation effort, of both the search direction and step, when the value of the objective function has not been reduced within an iteration level. The extension of the trust region is determined using a process similar to that described by More [7]. 
The algorithm presented above is applicable to the problems where the variables are not subject to restrictions. As a general rule, the regression variables (components' parameters or EOS coefficients) must satisfy restrictions of the inequality type:

$$
\xi_{1, \min } \leq \xi_{i} \leq \xi_{1, \max } ; i=1, n s ; n s \leq n r
$$

Such restrictions are required in order not to obtain physically.unacceptable variables and results values, as well as to maintain the variation trends of the pseudo-components' physical properties.

The following change of variable was suggested [4]:

$$
\xi_{i}=\xi_{i, \min }+\frac{\xi_{i, \max }-\xi_{i, \min }}{1+\xi_{\mathrm{i}, \max } \exp \left(-\mathrm{x}_{\mathrm{i}}\right)} ; \mathrm{i}=1, \mathrm{nr}
$$

to ensure that $\xi_{\mathrm{i}} \in\left(\xi_{\mathrm{i}, \min }, \xi_{\mathrm{i}, \max }\right)(\forall) \mathrm{x}_{\mathrm{i}} \in \mathbf{R}$. The $\xi=\xi(\mathrm{x})$ function has $\xi_{\mathrm{i}}=\xi_{\mathrm{i}, \min }$ and $\xi_{\mathrm{i}}=\xi_{\mathrm{i}, \max }$ as horizontal asymptotes to $-\infty$ and $+\infty$, respectively, and represents a strictly increasing function over $\mathrm{R}$. For the regression variables the values of the limits used by Coats and Smart [2] have been adopted.

Through these changes of variables, the constrained optimization problem with inequality type restrictions and with the $\xi_{\mathrm{i}}, \mathrm{i}=1, \mathrm{nr}$ variables was transformed into an unconstrained optimization problem, using the $\mathrm{x}_{\mathrm{i}}, \mathrm{i}=1$, nr variables, that can be solved with the Levenberg-Marquardt algorithm.

\section{THE GENERALIZED QUADRATURE METHOD FOR SEMICONTINUOUS THERMODYNAMICS}

The essential idea of describing the hydrocarbon mixtures composition based on continuous thermodynamics consists in the fact that discrete molar fractions, $x_{i}$, must be replaced with a continuous components' distribution. The distribution function, $\mathrm{F}(\mathrm{I})$ has as variable a certain parameter I characterizing the component, such as the effective carbon number (ECN), the molecular mass, etc., Owing to their simple forms, analytical forms, such as gamma, exponential or log-normal distributions can be used. These distributions can be defined over infinite or semi-infinite intervals of the characterization variable [8].

The distribution function can be used to model the repartition of components within the entire system or within part of the system. In the semi-continuous description, the molar fractions of some components (e.g. $\mathrm{C}_{1}-\mathrm{C}_{6}, \mathrm{CO}_{2}, \mathrm{~N}_{2}$ ) have discrete values, while the distribution of the molar fractions of heavy components $\left(\mathrm{C}_{7+}\right)$ is described in a continuous manner, with a distribution function.

The description based on the semi-continuous thermodynamics of the mixtures' composition in view of phase equilibrium calculation with EOS requires to go through the following sequential 5 steps:

1. Selection of the distribution function.

2. Determination of the distribution function parameters.

3. Selection of the pseudo-components, to allow their placement in the nodes of the Gauss type quadrature.

4. Assignment of critical parameters to the pseudo-components.

5. Studying the system with an EOS as if the system was characterized in a discrete manner.

The Gaussian quadrature represents an efficient method of integrating the functions by summing up a finite number of the function values (affected by weights) corresponding to specific integration variables denoted as the quadratures nodes. If the quadrature method is applied to the continuous thermodynamics, the phase equilibrium and the material balance are precisely satisfied for each quadrature node.

For a semi-continuous description, the normalizing condition and the $\mathrm{A}$ and $\mathrm{B}$ coefficients of the EOS are approximated through the quadrature method in the following manner:

$$
\begin{aligned}
& \sum_{i=1}^{n_{d}} z_{i}+\eta \sum_{p=1}^{m} w\left(I_{p}\right) F\left(I_{p}\right)=1 \\
& A \equiv \sum_{i=1}^{n_{d}} \sum_{j=1}^{n_{d}} z_{i} z_{j} \sqrt{A_{i} A_{j}}\left(1-C_{i j}\right)+2 \sum_{j=1}^{n_{d}} z_{j} \sqrt{A_{j}} \sum_{p=1}^{m} w\left(I_{p}\right) F\left(I_{p}\right) \sqrt{A\left(I_{p}\right)}+\sum_{p=1}^{m} \sum_{q=1}^{m} w\left(I_{p}\right) F\left(I_{p}\right) w\left(I_{q}\right) F\left(I_{q}\right) \sqrt{A\left(I_{p}\right) A\left(I_{q}\right)}(1 \\
& B \equiv \sum_{i=1}^{n_{d}} z_{j} B_{i}+\sum_{p=1}^{m} w\left(I_{p}\right) F\left(I_{p}\right) B\left(I_{p}\right)
\end{aligned}
$$

where $f\left(I_{p}\right)$ is the value of the function to be integrated in the quadrature nodes, $I_{p}$, and $w\left(I_{p}\right)$ is the weighting factor associated to the $I_{p}$ node, $\eta$ being the molar participation in the mixture of the continuous fraction ( $m$ pseudocomponents), and $n_{d}$ representing the number of discretely characterized components.

After having solved the quadrature (see Appendix), the nodes will determine the values of the pseudocomponents characterization variables I:

$$
\mathrm{I}_{\mathrm{i}}=\varphi\left(\mathrm{x}_{\mathrm{i}}\right) ; \mathrm{i}=1, \mathrm{~m}
$$

while the pseudo-components molar fractions will be obtained depending on the weights:

$$
\mathbf{z}_{\mathbf{i}}=\eta w_{\mathrm{i}} ; \mathbf{i}=1, \mathbf{m}
$$


Neither the form of the distribution function, nor the nature of the characterization variable will be involved in this method for solving the quadrature.

Cotterman and Prausnitz use a gamma distribution function, but the method for setting up the nodes and the (III-8) relation of [9] are not accurate. The above mentioned authors come back to the problem with an accurate solution of the quadrature [10], but limited only to semi-infinite distributions. The above-presented model is also applicable in the case of truncated distributions. The need and importance of truncating the distribution are discussed by Behrens and Sandler [8] who provide a complete solution of the quadrature for the exponential distribution.

\section{APPLICATIONS}

\section{a) The Pazanan system}

For the Pazanan (Iran) gas condensate system [11], after the adjustment of the BIC's, for a semicontinuous description of a 13-components mixture (three $C_{7+}$ pseudocomponents), the results obtained using the PREOS still show deviations from the experimental data. The composition of the $\mathrm{C}_{7+}$ fraction is represented in Figure 1, together with the exponential and gamma distribution (used here) functions.

The experimental data for which the regression was performed $(n e=12)$ are the following: the dew point pressure $\left(p_{d}\right)$, the $Z$ factor for gas at the dew point pressure $\left(Z_{d}\right)$, the liquid saturation $\left(S_{L}\right)$ and the gas compressibility factor $\left(Z_{\mathrm{v}}\right)$ for the 5 pressure stages of the $C V D$.

The regression variables are: $\Omega_{a}$ and $\Omega_{b}$ for methane and the pseudocomponents, and the BIC's between methane and the pseudo-components $(n=11)$. Application of the regression will lead to a significant reduction in the relative error for $\mathrm{S}_{\mathrm{L}}$, from around $19 \%$ to $0.4 \%$. The convergence was obtained through 8 iterations. Figures 2 and 3 show the experimental data and the results obtained before (equations PR and $P R 3$ ) and after the regression for $S_{L}$ and $Z_{V}$, respectively.

\section{b) Coats' near-critical system}

The near-critical gas condensate system presented by Coats [1] is described using 10 components, of which two are pseudocomponents (selected by assuming an exponential distribution of the components in the $\mathrm{C}_{7+}$ fraction). Further on, the results of application of the regression for two cases are presented.

CASE 1: The experimental data $(n e=11)$ are: $\mathrm{p}_{\mathrm{d}}, \mathrm{Z}_{\mathrm{d}}$ and $\mathrm{S}_{\mathrm{L}}$ at 9 CCE pressure stages and $\mathrm{n}=8$. Convergence was obtained through 9 iterations, and the value of the objective function was reduced from 2.882 to 0.106 . A very good agreement is noticed between calculated and experimental data in the vicinity of $S_{\text {Lmax }}$, but $S_{L}$ was under-estimatęd for low pressures. The $S_{L}(p)$ variation illustrated in Figure 4 is very close to that obtained by Coats (Figure 2 [1]). Though the relative volumes of the vapor phase were not requested as a target of regression, the closeness of their values to those of experimental data is quite remarkable (Figure 5). CASE 2 : From the set of experimental data considered under Case 1, the $\mathrm{S}_{\mathrm{L}}$ values for the last two pressure stages (ne-9) were excluded, while the weights were changed, so as to reduce the deviations over the entire pressure range. The results are presented in Figure $4\left(S_{L}\right) ; 7$ iterations were necessary to reduce the objective function from 0.2405 to 0.0232 .

c) The SPE3 system

The problem addressed by the third SPE (1987) comparative solutions project was the compositional simulation of gas recycling in a gas condensate reservoir [12]. A particular attention was given to the manner in which the participants ( 9 companies) solved the problem of matching the EOS results with PVT data.

The composition of the system is described in a semicontinuous manner, and the distribution of components heavier than heptane, has been modelled according to an exponential distribution which agrees with the experimental discrete description, as shown in Figure 6. Three pseudo-components were used.

The regression was applied for two situations considered in [12], namely:

CASE A: The experimental values are: $\mathrm{p}_{\mathrm{d}}, \mathrm{Z}_{\mathrm{d}}, \mathrm{S}_{\mathrm{L}}$ and $\mathrm{Z}_{\mathrm{V}}$ at the 5 pressures stages of the CVD (ne=12). The value of the objective function was reduced from 2.44 to $1.8310^{-3}$ through 7 iterations. The relative errors are: $1.12 \%$ for $S_{L}$ and $0.74 \%$ for $Z_{V}$. Figures 7 and 8 show the regression results for $S_{L}$, and for $Z_{V}$ respectively, and the cumulative of produced gas (molar percent).

$C A S E B$ : Besides the data required for the first case, it was requested to model the behaviour of mixtures made up of the gas condensate system and of an injection gas, present in the mixture in various ratios.

In addition to the data of the previous case, the set of experimental data comprised the dew point pressures of the mixtures corresponding to 4 participations of the injection gas (ne=16). The dew point pressures (relative error $=0.5 \%$ ) and the relative volumes (relative error $=1.38 \%$ ) for the swelling test are given in Figures 9 and 10, respectively. Convergence was obtained through 12 iterations.

In both cases, the agreement between the results and the experimental data is quite good (comparable or better than the results presented in [12], Figures 3, 5 and 6). The PREOS was used. 


\section{CONCLUSIONS}

1. An algorithm and the associated computer program were developed, to establish the agreement between the results of models based on EOS, and the PVT experimental data. The regression algorithm exhibits a high convergence speed and a remarkable lack of sensitiveness to the initial guesses.

2. A generalized quadrature method, applicable to any distribution function, as well as to truncated distribution has been developed in order to describe in a semicontinuous manner the composition of mixtures. 3. The algorithms have been successfully applied in the study of the behaviour of several gas condensate systems. This paper presents the results of three applications.

\section{REFERENCES}

1. Coats K.H. : Simulation of Gas Condensate Reservoir Performance, JPT (Oct. 1985), 1870-1885.

2. Coats K.H., Smart G.T. : Application of a Regression-Based EOS PVT Program to Laboratory Data, SPERE (May 1986), 277-299.

3. Agarwal R.K., Li Y.K., Nghiem L.X. : A Regression Technique With Dynamic Parameters Selection for Phase-Behavior Matching, SPERE(Feb. 1990), 115-120.

4. Nichita D. V.: Numerical Simulation of Gas-Condensate Reservoir Performances, Ph. D. dissertation, University of Ploiesti, 1996.

5. Nichita D.V., Cretu I., Minescu F: A New Method for Rapid Liquid-Vapor Phase Equilibria Calculation, Revista Română de Petrol, Vol 3. No. 4, Aug. 1996, 309-320.

6. Nichita D.V., Minescu F., Cretu I.: A New Reduced Flash Approach for Compositional Reservoir Simulation, The 8th International Scientific and Technical Conference, University of Mining \& Metallurgy, Krakow, 19-20 June 1997, Proceedings, Vol. 2, 305-327.

7. Moré J.J.: The Levenberg-Marquardt Algorithm: Implementation and Theory, Lecture Notes in Mathematics, No 630, Numerical Analysis, G. Watson (ed.), Springer-Verlag, NY (1978), 105-116.

8. Behrens R.A., Sandler S.I. : The Use of Semicontinuous Description To Model the $\mathbf{C}_{7+}$ Fraction in Equation of State Calculations, SPERE (Aug 1988), 1041-1047.

9. Cotterman R.L., Prausnitz J.M. : Flash Calculations for Continuous or Semicontinuous Mixtures Using an Equation of State, IEC PDD, 1986, 24, 434-443.

10. Cotterman R.L., Prausnitz J.M. : Comments on "Flash Calculations for Continuous or Semicontinuous Mixtures Using an Equation of State", IEC PDD, 1988, 25, 840-841.

11. Firoozabadi A., Hekim Y., Katz D.L. : Reservoir Depletion Calculations for Gas Condensates Using Extended Analyses in the Peng-Robinson Equation of State, Cdn. J. Chem. Eng. (Oct. 1978) 56, 610-615.

12. Kenyon D.E., Behie G.A.: Third SPE Comparative Solution Project: Gas Cycling of Retrograde Condensate Reservoirs, JPT (Aug. 1987), 981-997.

\section{APPENDIX}

In order to solve the quadrature it should be first noted that the integrals involved in the molar balance relation and in the expressions of the state equation coefficients will have the following form:

$$
\mathrm{H}=\int_{A}^{B} \mathrm{D}(\mathrm{l}) \mathrm{p}(\mathrm{l}) \mathrm{dl} \quad \text { with } \mathrm{A} \leq \mathrm{I} \leq \mathrm{B} \leq \infty
$$

where $p(I)$ is the weighting function associated to the utilized distribution function, and orthogonal to it over the $[A, B]$ interval. If a change of variable is introduced:

$$
I=\varphi(x)
$$

selected in such a manner that $\varphi(0)=A$, where the $\varphi$ function is a bijective application (depending on the distribution function), then the (Al) integral will become:

$$
H=\int_{0}^{c} D(x) p(x) d x \cong \sum_{i=1}^{m} D\left(x_{i}\right) w_{i}
$$

where $c=\varphi^{-1}(B)$. The $x_{i}$ nodes are the roots of the equation:

$$
Q_{m}=\left|\begin{array}{cccc}
x^{m} & x^{m-1} & \ldots & 1 \\
M_{2 m-1} & M_{2 m-2} & \cdots & M_{m-1} \\
\cdots & \cdots & \cdots & \ldots . \\
M_{m} & M_{m-1} & \cdots & M_{0}
\end{array}\right|=0
$$

where:

$$
M_{k}=\int_{0}^{c} e^{-s} s^{k} d s ; k=0,2 m-I
$$

The $\mathrm{Q}_{\mathrm{m}}(\mathrm{x})$ polynomials have real and distinct roots, comprised between 0 and $\mathrm{c}$. For $\mathrm{c}=\infty$ and $\mathrm{p}(\mathrm{x})=\mathrm{e}^{-\mathrm{x}} \mathrm{x}^{\alpha}$, $\mathrm{Q}_{\mathbf{m}}(\mathrm{x})$ are Laguerre polynomials. The quadrature weights are given by the solution of the linear system:

$$
\sum_{j=1}^{m} w_{i} x_{j}^{k}=\int_{0}^{c} p(s) s^{k} d s ; k=0, m-1 ; j=1, m
$$




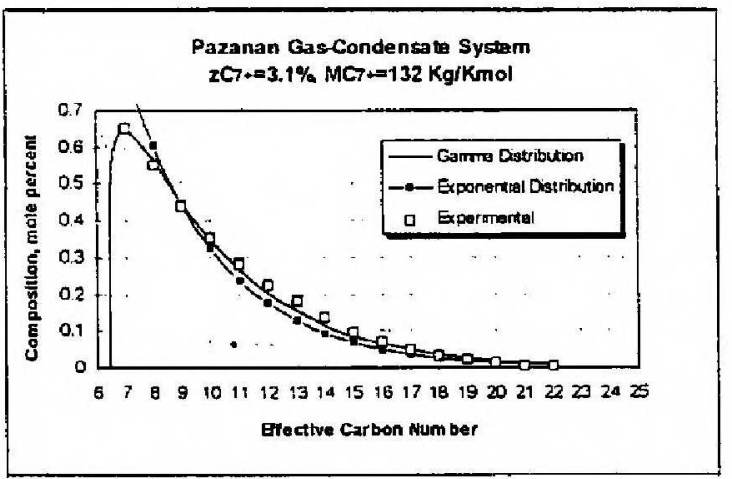

Fig. 1 - Composition of $C_{7+}$ fraction. Pazanan gas condensate system.

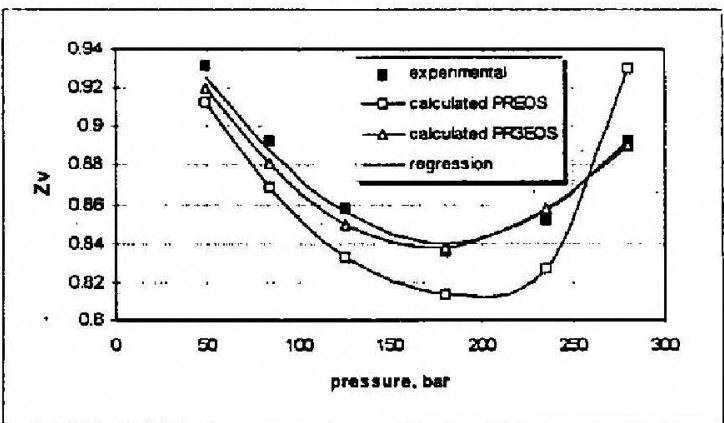

Fig. 3 - Regression for Pazanan system. Vapor Z-factor. CVD at $\mathrm{T}=355.7 \mathrm{~K}$

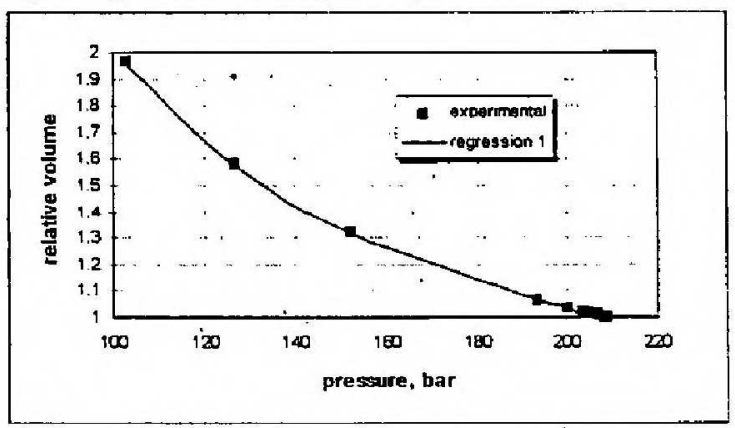

Fig. 5 - Regression for Coats system. Relative volume. CCE at $T=436 \mathrm{~K}$.

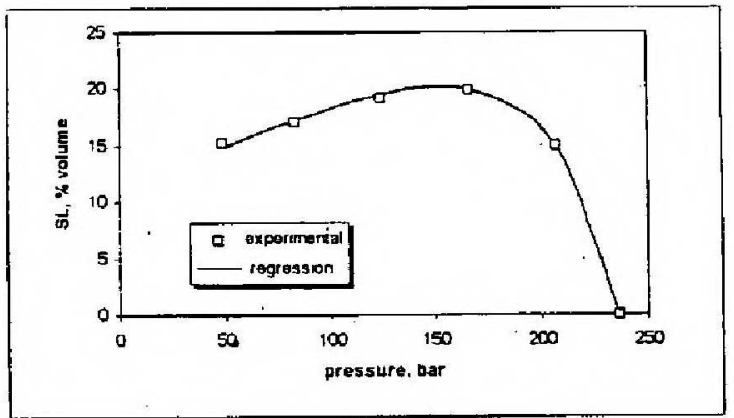

Fig. 7 - Results of regression (Case A) for SPE3 system. Liquid saturatuon. $\mathrm{CVD}$ at $\mathrm{T}=366.5 \mathrm{~K}$

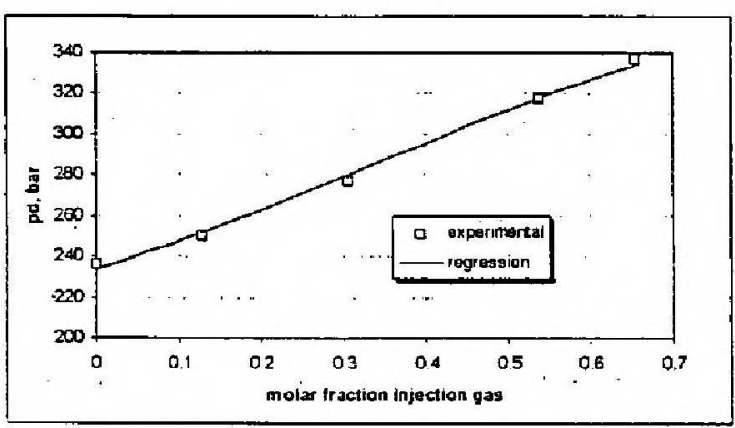

Fig. 9 - Results of regression (Case B) for SPE3 system Dew point pressures for the swelling test.

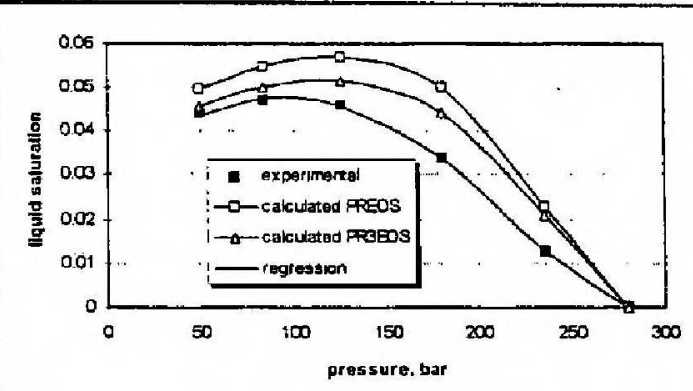

Fig. 2 - Regression for Pazanan system. Liquid saturation. CVD at $\mathrm{T}=355.7 \mathrm{~K}$,

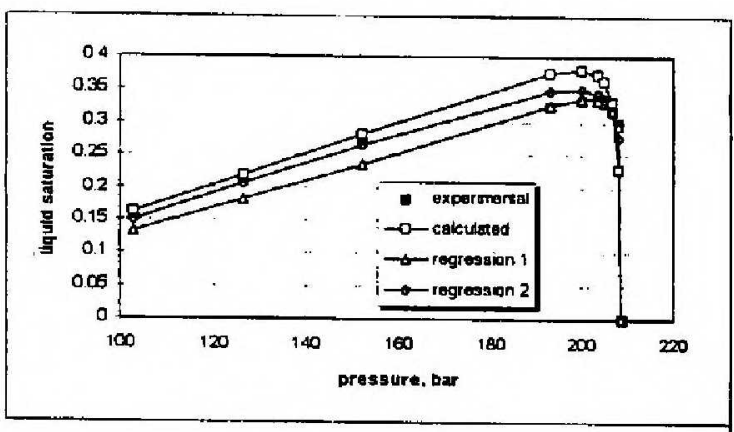

Fig 4 - Regression for Coats system. Liquid saturacion. CCE at $T=436 \mathrm{~K}$.

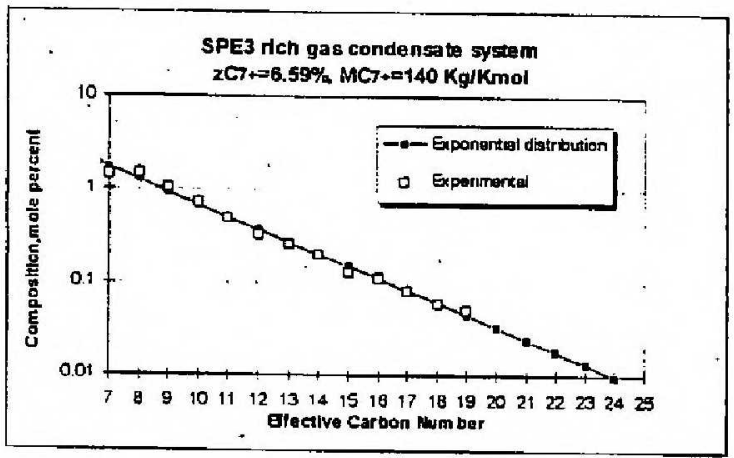

Fig. 6 - Composition of $\mathrm{C} 7+$ fraction. SPE3 gas condensate systern.

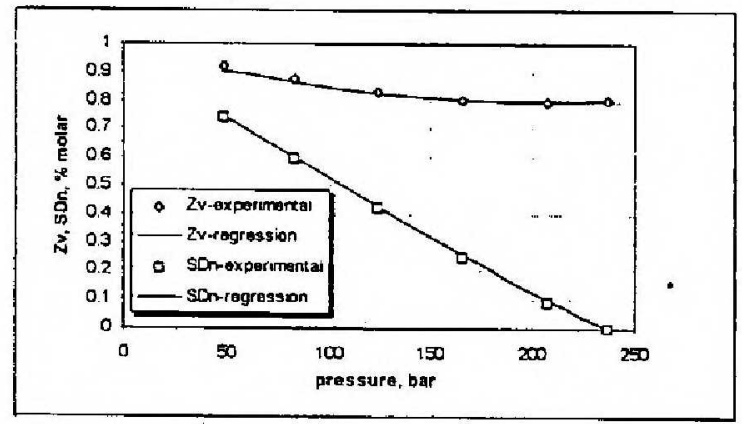

Fig. 8 - Results of regression (Case A) for SPE3 system. Vapor $Z$ factor and cumulative of produced gas ( $\mathrm{SD} n)$. $\mathrm{CVD}$ at $\mathrm{T}=366.5 \mathrm{~K}$.

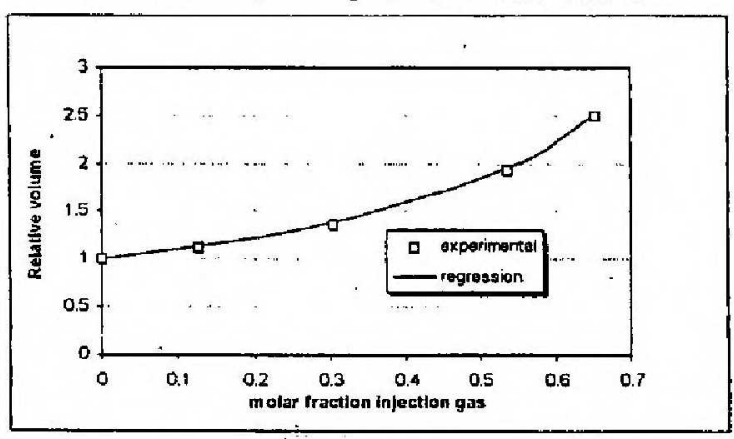

Fig. 10 - Results of regression (Case B) for SPE3 system. Relative volumes for the swelling test. 